\title{
Detecting Structure in Diffusion Tensor MR Images
}

\author{
K. Krishna Nand ${ }^{1}$, Rafeef Abugharbieh ${ }^{1}$, Brian G. Booth ${ }^{2}$, and Ghassan Hamarneh ${ }^{2}$ \\ ${ }^{1}$ Biomedical Signal and Image Computing Lab, University of British Columbia \\ ${ }^{2}$ Medical Image Analysis Lab, School of Computing Science, Simon Fraser University \\ $\{k k r i s h n a, r a f e e f\}$ eece.ubc.ca, \{bgb2, hamarneh\}@sfu.ca
}

\begin{abstract}
We derive herein first and second-order differential operators for detecting structure in diffusion tensor MRI (DTI). Unlike existing methods, we are able to generate full first and second-order differentials without dimensionality reduction and while respecting the underlying manifold of the data. Further, we extend corner and curvature feature detectors to DTI using our differential operators. Results using the feature detectors on diffusion tensor MR images show the ability to highlight structure within the image that existing methods cannot.
\end{abstract}

\section{Introduction}

Feature detection is a core component in most image processing and analysis tasks with ubiquitous applications ranging from data registration to object segmentation, classification, and recognition. Of the multitude of feature detectors available today, those that are widely used typically involve first and second order spatial differential operators [9]18]. We desire the ability to use such feature detectors to highlight anatomical structure in diffusion tensor images (DTI). To date, only a few attempts have been made to develop differential operators for such manifold-valued image data. Table 1 summarizes the current state-of-the-art and highlights a conspicuous gap in the current methodology: the ability of current differential operators to respect the manifold-valued nature of diffusion tensor data.

Table 1. Summary of existing and proposed work in differential-based feature detection for scalar and manifold-valued data. The manifold of symmetric second-order positive definite tensors is represented as $\operatorname{SPD}(3)$. Methods listed for $\mathbb{R}^{N}$ also apply to $\mathbb{R}^{3}$. Note our contributions in the lower-right of the table. They are derived in Equations (1-3).

\begin{tabular}{|c|c|c|c|}
\hline $\begin{array}{c}\text { Data } \\
\text { Dimensionality }\end{array}$ & \begin{tabular}{|c|} 
First-Order Differentials \\
Magnitude Only $\mid$ Full Differential \\
\end{tabular} & \multicolumn{2}{|c|}{$\begin{array}{c}\text { Second-Order Differentials } \\
\text { Magnitude Only|Full Differential }\end{array}$} \\
\hline $\mathbb{R}$ & $\begin{array}{c}\text { Edge Detection }[5] \\
\text { Corner Detection }[18]\end{array}$ & \multicolumn{2}{|c|}{$\begin{array}{l}\text { Frangi et al. Curvature [9] } \\
\text { Curvature Magnitude [12] }\end{array}$} \\
\hline $\mathbb{R}^{3}$ & MPT Projection [20] & \multicolumn{2}{|c|}{ Quaternions [19] } \\
\hline $\mathbb{R}^{N}$ & $\begin{array}{c}\text { Channel Normalization [8] } \\
\text { Third-Order Tensors [17] } \\
\text { Structure Tensor [6] }\end{array}$ & $\begin{array}{l}\text { Directional } \\
\text { Derivative of } \\
\text { Gradient [1] }\end{array}$ & $\begin{array}{c}\text { Weighted } \\
\text { Channel Av- } \\
\text { eraging }[13]\end{array}$ \\
\hline $\operatorname{SPD}(3)$ & \begin{tabular}{|c|c|} 
Distance Metrics & Our Contribution: \\
{$[2]$} & Equations (1]-2] \\
\end{tabular} & \multicolumn{2}{|c|}{$\begin{array}{c}\text { Our Contribution: } \\
\text { Equation }\end{array}$} \\
\hline
\end{tabular}

G. Fichtinger, A. Martel, and T. Peters (Eds.): MICCAI 2011, Part II, LNCS 6892, pp. 90-97, 2011.

(C) Springer-Verlag Berlin Heidelberg 2011 
In this paper, we derive first and second order differential operators for diffusion tensor fields that respect the manifold of second-order tensors. Using these differential operators, we extend the Harris and Shi-Tomasi corner detectors to DTI. We further construct curvature based features detectors to detect tube-like and sheet-like structures in DTI. These features allow us to capture structural information within a DT image that has previously not been explored. We note here that our approach to detecting these structural features is different from other approaches [11 15|21] in that we work with more fundamental operators of low-level computer vision as opposed to higher level characterization of the data.

\section{Methods}

\subsection{First Order Operators}

We begin our exposition by deriving the first-order differential operator of a tensor field. To ensure that we respect the manifold of second-order tensors, we employ the Log-Euclidean (LE) mapping proposed in [2] to map the space of symmetric second order positive definite tensors, $\operatorname{SPD}(3)$, into a vector space. Let $\boldsymbol{D}(\boldsymbol{x})$ be a 3D DT image indexed by $\boldsymbol{x}=\left[\begin{array}{lll}x_{1} & x_{2} & x_{3}\end{array}\right]$. Then the LE mapping gives $\boldsymbol{L}(\boldsymbol{x})=\operatorname{logm}(\boldsymbol{D}(\boldsymbol{x}))$, where $\boldsymbol{L}(\boldsymbol{x})$ is the LE tensor and $\operatorname{logm}(\cdot)$ is the matrix logarithm.

Let $\boldsymbol{J}(\boldsymbol{x})$ denote the Jacobian matrix, given by $J_{i j}=\frac{\partial L_{i}}{\partial x_{j}} \cdot \boldsymbol{J}(\boldsymbol{x})$ generalizes the gradient of a scalar field to the derivatives of a vector field. We define the $3 \times 3$ symmetric positive semi-definite matrix $S(x)=\boldsymbol{J}(x)^{T} \boldsymbol{J}(x)$. The matrix $S(x)$, referred to as the structure tensor, measures the directional dependence of total change. Let $\lambda_{1}^{S} \geq \lambda_{2}^{S} \geq \lambda_{3}^{S}$ denote the eigenvalues of $\boldsymbol{S}(\boldsymbol{x})$ and let $\hat{\boldsymbol{e}}_{\boldsymbol{i}}^{S}$ be the eigenvector corresponding to $\lambda_{i}^{S}$. The squared Euclidean norm of $d \boldsymbol{L}(\boldsymbol{x})$ can be written in terms of $\boldsymbol{S}(\boldsymbol{x})$ as $\|d \boldsymbol{L}(\boldsymbol{x})\|^{2}=(d \boldsymbol{x})^{T} \boldsymbol{S}(\boldsymbol{x}) d \boldsymbol{x}$. This positive definite quadratic form is called the first fundamental form. For a unit vector $\boldsymbol{n}, A(\boldsymbol{x})=\boldsymbol{n}^{T} \boldsymbol{S}(\boldsymbol{x}) \boldsymbol{n}$ measures the rate of change of the image in the direction $\boldsymbol{n}$ and is referred to as the squared local contrast [6]. It is maximum when $\boldsymbol{n}$ is in the direction of the eigenvector corresponding to the largest eigenvalue of $\boldsymbol{S}(\boldsymbol{x})$. As a result, this eigenvector is used as the gradient direction $\hat{\boldsymbol{g}}(\boldsymbol{x})$. To resolve the sign ambiguity of the eigenvector, we use a voting approach across channels. The gradient magnitude $|\boldsymbol{g}(\boldsymbol{x})|$ is set to be the square root of the corresponding eigenvalue of $\boldsymbol{S}(\boldsymbol{x})$. The resulting gradient vector is then given by:

$$
\boldsymbol{g}(\boldsymbol{x})=\sqrt{\lambda_{1}^{S}} \hat{e}_{1}^{S} .
$$

A closed-form solution for (1) in terms of $\boldsymbol{S}(\boldsymbol{x})$ can be derived but is not presented here due to space limitations.

An averaged version of $\boldsymbol{S}$ around a local neighborhood, $\overline{\boldsymbol{S}}$, allows the integration of first order information from the neighborhood and a more stable numerical derivation. We call $\overline{\boldsymbol{S}}$, the log-Euclidean structure tensor and construct it as:

$$
\bar{S}_{i k}\left(\sigma_{I}, \boldsymbol{x}\right)=\boldsymbol{\omega}_{\sigma_{I}}(\boldsymbol{x}) * \sum_{j} \frac{\partial L_{j}}{\partial x_{i}} \frac{\partial L_{j}}{\partial x_{k}},
$$

where $\boldsymbol{\omega}_{\sigma_{I}}$ is a Gaussian window with standard deviation $\sigma_{I}$ and $L_{j}$ is the $j^{\text {th }}$ component of $\boldsymbol{L}(\boldsymbol{x})$. 


\subsection{Second Order Operators}

The Hessian matrix is a symmetric matrix consisting of second order partial derivatives. It describes the local second order structure around each pixel in an image. We derive two forms of the Hessian matrix. The first, $\boldsymbol{H}_{1}$, is based on simple weighted averaging of the Hessian matrices of each component of $\boldsymbol{L}(\boldsymbol{x})$, as done for color images in [13]. Our second formulation is based on the gradient vector we derived earlier in (1). Given $\boldsymbol{g}(\boldsymbol{x})=\left[\begin{array}{lll}g_{x_{1}} & g_{x_{2}} & g_{x_{3}}\end{array}\right]^{T}$ has been computed at every location $\boldsymbol{x}$ of the DT image, the gradient vector field $\boldsymbol{g}$ can be considered as a multivalued function with the mapping $\Omega_{g}: \mathbb{R}^{3} \rightarrow \mathbb{R}^{3}$. Using this context, we compute the derivative of the gradient vector field via the Jacobian matrix, $\boldsymbol{G}(\boldsymbol{x})$, as $G_{i j}=\frac{\partial g_{i}}{\partial x_{j}}$. $\boldsymbol{G}(\boldsymbol{x})$ encodes second order information of $\boldsymbol{L}(\boldsymbol{x})$ and, in scalar images, $\boldsymbol{G}(\boldsymbol{x})$ represents the Hessian matrix. However in multivalued images, $\boldsymbol{G}(\boldsymbol{x})$ is generally non-symmetric. Our second formulation for the Hessian matrix $\boldsymbol{H}_{\mathbf{2}}$ in multivalued images is thus given by:

$$
H_{2}(x)=\frac{G(x)+G(x)^{T}}{2},
$$

which is the symmetric part of $\boldsymbol{G}(\boldsymbol{x})$ and is the best $L_{2}$ approximation to $\boldsymbol{G}(\boldsymbol{x})$ from the set of symmetric matrices [3]. Our first order (112) and second order (3) differential operators can easily be extended to different scales.

\subsection{Feature Detectors}

Our proposed differential operators fit naturally into existing feature detectors and can thus be effectively used to detect structure in DTI. Here we provide four examples from a potentially long list of feature detectors that can incorporate our differential operators. Using our first order differential in (2), we detect corners in DT images using the popular Harris $\left(C_{H}\right)$ [16] and Shi-Tomasi $\left(C_{S T}\right)$ [18] corner detectors which we define as:

$$
C_{H}=\frac{\operatorname{det}(\overline{\boldsymbol{S}})}{\operatorname{tr}(\overline{\boldsymbol{S}})+\epsilon}
$$

$$
C_{S T}=\min \left(\lambda_{1}^{\bar{S}}, \lambda_{2}^{\bar{S}}, \lambda_{3}^{\bar{S}}\right)
$$

where $\operatorname{det}(\overline{\boldsymbol{S}})$ is the determinant of $\overline{\boldsymbol{S}}, \operatorname{tr}(\overline{\boldsymbol{S}})$ the trace of $\overline{\boldsymbol{S}}$ and $\lambda_{1}^{\overline{\boldsymbol{S}}} \geq \lambda_{2}^{\overline{\boldsymbol{S}}} \geq \lambda_{3}^{\overline{\boldsymbol{S}}}$ are the eigenvalues of $\overline{\boldsymbol{S}}$.

Curvature cues can also be obtained from our Hessian matrix derived in (3). As examples, we extend the vesselness filter of [9] and the sheetness filter of [7] to detect tube-like and sheet-like fiber tracts in DT11. Our tubular-ness and sheet-ness measures are thus defined as:

$$
\begin{aligned}
C_{\text {tube }} & =\left(1-\exp \left(-\frac{\mathcal{R}_{A}^{2}}{2 \alpha^{2}}\right)\right)\left(\exp \left(-\frac{\mathcal{R}_{B}^{2}}{2 \beta^{2}}\right)\right)\left(1-\exp \left(-\frac{\mathcal{S}^{2}}{2 c^{2}}\right)\right), \\
C_{\text {sheet }} & =\left(\exp \left(-\frac{\mathcal{R}_{A}^{2}}{2 \alpha^{2}}\right)\right)\left(1-\exp \left(-\frac{\mathcal{R}_{D}^{2}}{2 \eta^{2}}\right)\right)\left(1-\exp \left(-\frac{\mathcal{S}^{2}}{2 c^{2}}\right)\right),
\end{aligned}
$$

\footnotetext{
${ }^{1}$ Here, it is important to note that tube-like and sheet-like refer to the structural characteristics of the DT image and not to the diffusion tensors.
} 
where $C_{\text {tube }}$ and $C_{\text {sheet }}$ measure how tubular and sheet-like the structure is, $\mathcal{R}_{A}=$ $\left|\lambda_{2}^{\boldsymbol{H}}\right| /\left|\lambda_{1}^{\boldsymbol{H}}\right|, \mathcal{R}_{B}=\left|\lambda_{3}^{\boldsymbol{H}}\right| / \sqrt{\left|\lambda_{1}^{\boldsymbol{H}} \lambda_{2}^{\boldsymbol{H}}\right|}, \mathcal{R}_{D}=\left|\left(2\left|\lambda_{1}^{\boldsymbol{H}}\right|-\left|\lambda_{2}^{\boldsymbol{H}}\right|-\left|\lambda_{3}^{\boldsymbol{H}}\right|\right)\right| /\left|\lambda_{1}^{\boldsymbol{H}}\right|$ and $\mathcal{S}=\sqrt{\left(\lambda_{1}^{\boldsymbol{H}}\right)^{2}+\left(\lambda_{2}^{\boldsymbol{H}}\right)^{2}+\left(\lambda_{3}^{\boldsymbol{H}}\right)^{2}}$. The measures $\mathcal{R}_{A}, \mathcal{R}_{B}$ and $\mathcal{S}$ are used to penalize sheet-like structures, blob-like structures and noise, respectively. $\mathcal{R}_{D}$ is used to penalize tube like and blob like structures. These terms are computed from the eigenvalues $\lambda_{1}^{\boldsymbol{H}} \geq \lambda_{2}^{\boldsymbol{H}} \geq \lambda_{3}^{\boldsymbol{H}}$ of the Hessian matrix in (3). The constants $\alpha, \beta, \eta$ and $c$ control the sensitivity of $C_{\text {tube }}$ and $C_{\text {sheet }}$ to each term.

\section{Results}

We present results of the proposed differential operators and feature detectors on synthetic and real DT data. The real DT data consisted of 24 images of normal adult brains, of which, 12 were taken from the John Hopkins LBAM [14] database and 12 from the MIDAS database [4]. In our experiments, the constants $\alpha, \beta$ and $\eta$ were set to 0.5 (as suggested in [7]

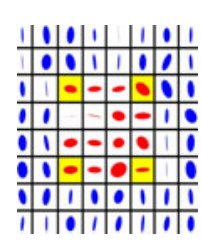

(a)Corner Phantom

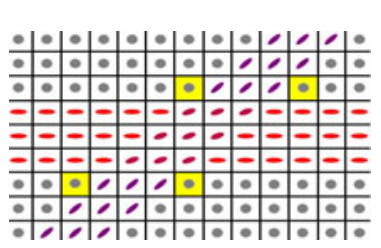

(b) Crossing Phantom

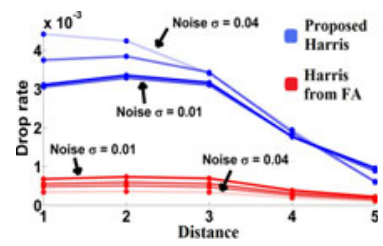

(c) Drop rate of Harris response vs. distance

Fig. 1. Results of the proposed Harris and Shi-Tomasi corner detectors on synthetic data. (a) and (b): two synthetic slices with the ground truth corner locations shown in yellow. (c): the drop rate of the Harris feature response as a function of distance from the ground truth corners for different levels of noise (standard deviation of noise ranging from 0.01 to 0.04 ). Results are shown for both our method and the approach in [16] on the FA maps. Note the response of our method falls rapidly for all levels of noise, demonstrating localization accuracy.

Figure 1 shows the proposed Harris and Shi-Tomasi corner detector results on synthetic data. The conventional scalar Harris and Shi-Tomasi features obtained from the fractional anisotropy (FA) maps are used for comparison. Figure 1(a) shows a noisy image where the tensors are pointing horizontally in the foreground (colored in red) while tensors point vertically in the background (colored in blue). Figure 1(b) shows an example of two fibers crossing each other. The ground truth corner points are shown in yellow. Varying levels of noise were added to these images using the method in [10]. We show in Figure 1(c) the drop rate in the feature response as a function of distance to the nearest ground truth corner point in the examples in Figures 1(a) and 1(b). Our results demonstrate how the response of the proposed Harris detector falls very quickly for all levels of noise when compared to the response of the scalar Harris detector on FA as we move away from the ground truth corners. We also observed consistent stronger response to corners for our proposed Harris detector compared to the approach based on 


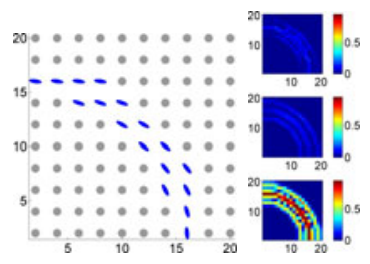

(a) Bent fiber tract (b)

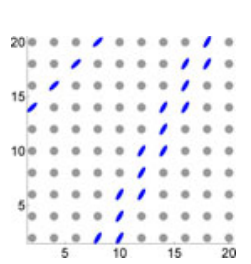

(c) Linear fibers

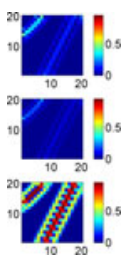

(d)

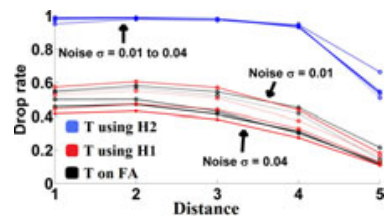

(e) Drop rate of tube-ness response vs. distance

Fig. 2. Tubular-ness results for synthetic $2 \mathrm{D}$ slices. Figures (a) and (c) show a bent fiber tract and linear fiber tracts oriented in different angles, respectively. Figures (b) and (d) show the corresponding tubular-ness responses obtained using Hessian from FA (top), $\boldsymbol{H}_{\mathbf{1}}$ (middle) and $\boldsymbol{H}_{2}$ (bottom). Note that tubular-ness obtained using $\boldsymbol{H}_{2}$ gives the strongest response in both cases. Figure (e) shows the drop of the filter's response as a function of distance to the ground truth medial of the tube. Note that even when noise is high, tubular-ness using $\boldsymbol{H}_{\mathbf{2}}$ decreases more rapidly than tubular-ness using $\boldsymbol{H}_{1}$ or FA, indicating greater localization ability.

the FA maps. A similar behavior was also observed for the Shi-Tomasi detector (figure not shown due to space constraints). This rapid decrease in response for our method indicates its greater ability to localize the corner points in various noise conditions.

Figure 2 shows results of tubular-ness filter on synthetic data. The tubular-ness obtained from the corresponding FA map using the scalar version of the filter in [9] is included for comparison. Figure 2(a) shows a bent fiber tract and figure 2(c) shows linear fiber tracts oriented along different angles. Figures 2(b) and 2(d) show the corresponding tubular-ness results. We observe that tubular-ness using $\boldsymbol{H}_{\mathbf{2}}$ gives the highest response in both cases. In figure 2(e), we add noise to these images and, as before, we measure the rate of decrease of the filter's response as a function of distance to the ground truth medial of the tube. We observe that tubular-ness using $\boldsymbol{H}_{\mathbf{2}}$ falls more rapidly when compared to the other filters, even when the noise is high. Again, this result illustrates our method's greater localization ability.

To quantify the information captured by the differential operators, we compute various norms of our structure tensor, gradient vector and Hessian matrices for twelve adult brain DTI from the LBAM database. Histograms of these norms are shown in Figure 3 for the proposed methods as well as those obtained from the FA map. Note that the norms of our proposed differential operators are spread over larger values than the norms of the differentials obtained from the FA maps. This result stems directly from avoiding the dimensionality reduction of the tensor data to FA values. Also note that $\boldsymbol{H}_{\mathbf{2}}$ captures a greater amount of differential information than the other two Hessian approaches as $\boldsymbol{H}_{\mathbf{2}}$ works with the whole tensor and not its individual channels or FA.

We applied our corner and tube detectors to 24 real adult brain DTI. Figure 4 shows a representative result for the corner detectors for both our method and the conventional scalar Harris [16] and Shi-Tomasi [18] features obtained from the FA maps. First, we observe that the feature response of our approach is stronger than the feature response from the FA-based approach by three orders of magnitude. Secondly our approach generates a more distinctive response to the corners of both the Genu and Splenium of the 


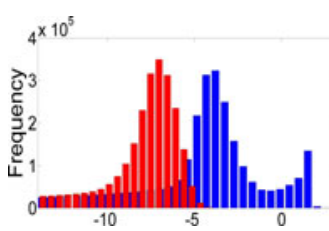

(a) Log Frobenius norm of ST

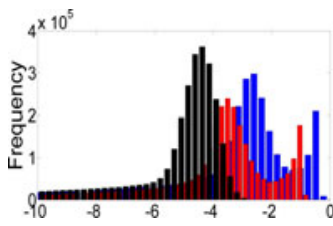

(e) Log Frobenius norm of $\mathrm{H}$

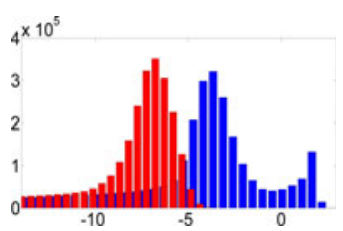

(b) Log row sum norm of ST

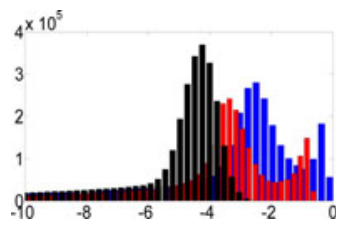

(f) Log row sum norm of $\mathrm{H}$

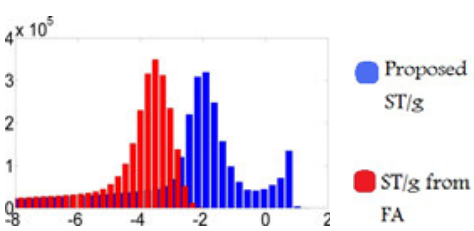

(c) Log L2 norm of g

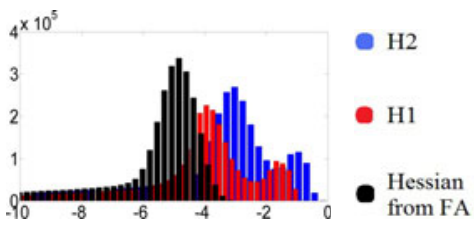

(g) Log max norm of $\mathrm{H}$

Fig. 3. Histograms of various norms of the structure tensor, gradient vector and Hessian matrix (abbreviated in the figure as ST, g and H, respectively) for twelve adult brain DT images from the LBAM database. Shown are results for the proposed methods and FA-based feature detection. Note the greater norm of our proposed differential operators compared to FA-based approaches, demonstrating that we are capturing more differential information.

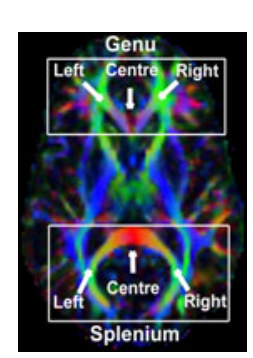

(a) Color FA

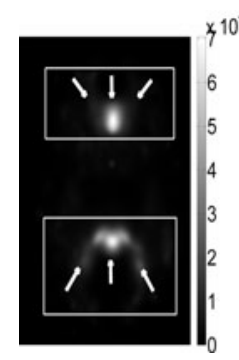

(b) Harris from FA

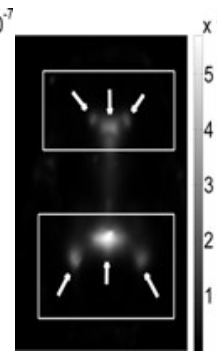

(c) Proposed Harris

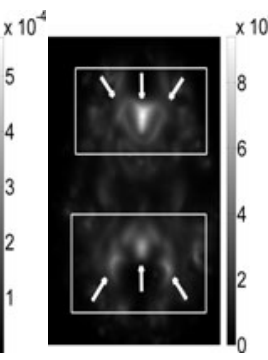

(d) Shi-Tom. on FA

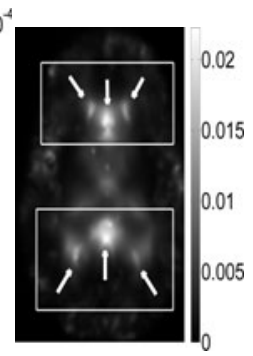

(e) Proposed Shi-Tom.

Fig. 4. Representative result for the Harris and Shi-Tomasi corner detectors on an adult brain DT image. Shown are (a) the color FA map, (b) Harris features from the FA map, (c) Harris features using our method, (d) Shi-Tomasi features from the FA map, and (e) Shi-Tomasi features using our method. Note that since the responses of the FA-based detectors are extremely weak when compared to the responses of our proposed detectors, they are shown with different intensity scales. Also note the clearer response to the corners of the Genu and Splenium using our method.

Corpus Callosum than the FA-based detectors. These results were consistently observed throughout the datasets.

Finally, Figure 5 shows representative tubular-ness and sheet-ness results. Note that tubular tracts such as the Cingulum and Fornix are well detected using $\boldsymbol{H}_{\mathbf{2}}$. Sheet-like tracts such as the Inferior Longitudinal Fasciculus, Corpus Callosum and Corticospinal Tract are also better detected using $\boldsymbol{H}_{2}$ compared to $\boldsymbol{H}_{1}$ and FA-based features. The FA-based features fail to detect structures in most cases. These results were consistently observed throughout the datasets. 

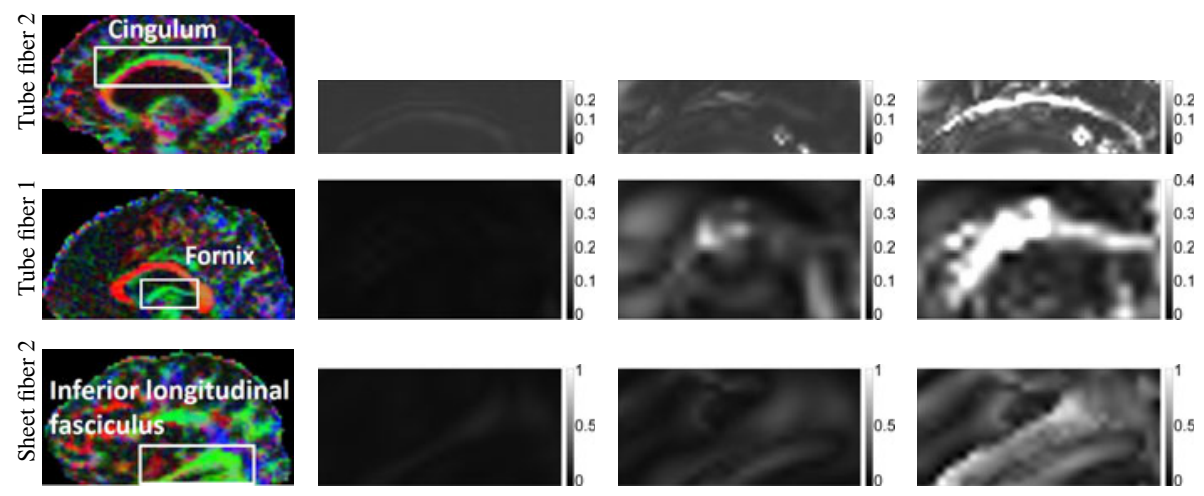

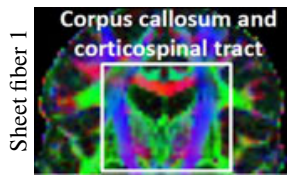

(a) Color FA

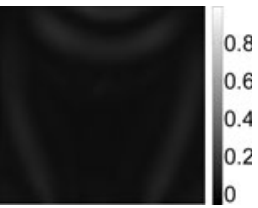

(b) Hessian from FA

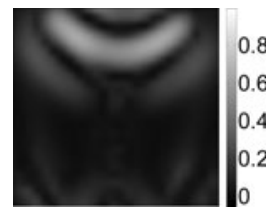

(c) $H_{1}$

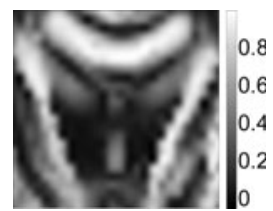

(d) $\boldsymbol{H}_{2}$

Fig. 5. Representative results for the tubular-ness and sheet-ness filters on adult brain DTI data. Shown from top to bottom are two results for tubular-ness and two results for sheet-ness. Shown from left to right are (a) color FA image, (b) results obtained using [79] on FA, (c) results obtained using $\boldsymbol{H}_{1}$ and (d) results obtained using $\boldsymbol{H}_{2}$. Note the identified tubular tracts (Cingulum, Fornix) and the identified sheet-like tracts (Inferior Longitudinal Fasciculus, Corpus Callosum, Corticospinal Tract) are better detected using $\boldsymbol{H}_{\mathbf{2}}$ than with $\boldsymbol{H}_{\mathbf{1}}$ and FA based method.

\section{Conclusion}

We derived novel first and second order differential operators for DTI. Unlike existing state-of-the-art, our operators respect the manifold of symmetric second-order tensors through the use of the log-Euclidean mapping. We further show how our differential operators can be naturally incorporated into various feature detectors in order to find structure in diffusion tensor images that, to date, has not been possible. We extend the Harris and Shi-Tomasi corner detectors to DTI and show that our approach better distinguishes corners in DTI data and is more robust to noise. We also extend the vesselness filter of [9] and the sheetness filter of [7] to detect tube-like and sheet-like structures. We show that our methods better detect these tube-like and sheet-like structures in DTI data and are more robust to noise than existing methods. We believe that our derived low-level operators and image features are very versatile and will be of great advantage to classification, registration, and segmentation of DTI data.

This work was supported in part by NSERC and the Institute for Computing, Information and

Cognitive Systems (ICICS) at UBC. 


\section{References}

1. Alshatti, W., Lambert, P.: Using eigenvectors of a vector field for deriving a second directional derivative operator for color images. In: Chetverikov, D., Kropatsch, W.G. (eds.) CAIP 1993. LNCS, vol. 719, pp. 149-156. Springer, Heidelberg (1993)

2. Arsigny, V., Fillard, P., Pennec, X., Ayache, N.: Log-Euclidean metrics for fast and simple calculus on diffusion tensors. Magnetic Resonance in Medicine 56(2), 411-421 (2006)

3. Bailey, R.A., Gower, J.C.: Approximating a symmetric matrix. Psychometrika 55(4), 665675 (1990)

4. Bullitt, E., Smith, J., Lin, W.: Designed database of MR brain images of healthy volunteers, http: / / www. insight-journal.org/midas/community/view/21 (accessed May 2010)

5. Canny, J.: A computational approach to edge detection. IEEE Trans. Pattern Anal. Mach. Intell. 8(6), 679-714 (1986)

6. Cumani, A.: Edge detection in multispectral images. Graphical Models and Image Processing 53(1), 40-51 (1991)

7. Descoteaux, M., Audette, M., Chinzei, K., Siddiqi, K.: Bone enhancement filtering: Application to sinus bone segmentation and simulation of pituitary surgery. In: Duncan, J.S., Gerig, G. (eds.) MICCAI 2005. LNCS, vol. 3749, pp. 9-16. Springer, Heidelberg (2005)

8. Evans, A.N., Liu, X.U.: A morphological gradient approach to color edge detection. IEEE Trans. on Image Processing 15(6), 1454-1463 (2006)

9. Frangi, A.F., Niessen, W.J., Vincken, K.L., Viergever, M.A.: Multiscale vessel enhancement filtering. In: Wells, W.M., Colchester, A.C.F., Delp, S.L. (eds.) MICCAI 1998. LNCS, vol. 1496, pp. 130-137. Springer, Heidelberg (1998)

10. Frindel, C., Robini, M., Croisille, P., Zhu, Y.-M.: Comparison of regularization methods for human cardiac diffusion tensor MRI. Medical Image Analysis 13(3), 405-418 (2009)

11. Goodlett, C.B., Fletcher, P.T., Gilmore, J.H., Gerig, G.: Group analysis of DTI fiber tract statistics with application to neurodevelopment. NeuroImage 45(1), 133-142 (2009)

12. Kindlmann, G., Tricoche, X., Westin, C.-F.: Delineating white matter structure in diffusion tensor MRI with anisotropy creases. Medical Image Analysis 11(5), 492-502 (2007)

13. Ming, A., Ma, H.: A blob detector in color images. In: CIVR 2007, pp. 364-370. ACM, New York (2007)

14. Mori, S.: John Hopkins Medical Institute: Laboratory of Brain Anatomical MRI, in vivo human database, http: / / lbam.med.jhmi.edu/(accessed February 2009)

15. Niethammer, M., Zach, C., Melonakos, J., Tannenbaum, A.: Near-tubular fiber bundle segmentation for diffusion weighted imaging: Segmentation through frame reorientation. NeuroImage 45(1), 123-132 (2009)

16. Noble, J.A.: Finding corners. Image and Vision Computing 6(2), 121-128 (1988)

17. Pajevic, S., Aldroubi, A., Basser, P.J.: A continuous tensor field approximation of discrete DT-MRI data for extracting microstructural and architectural features of tissue. Journal of Magnetic Resonance 154(1), 85-100 (2002)

18. Shi, J., Tomasi, C.: Good features to track. In: CVPR 1994, pp. 593-600 (1994)

19. Shi, L., Funt, B., Hamarneh, G.: Quaternion color curvature. In: IS\&T CIC 2008, pp. 338341 (2008)

20. Tsai, P., Chang, C.-C., Hu, Y.-C.: An adaptive two-stage edge detection scheme for digital color images. Real-Time Imaging 8(4), 329-343 (2002)

21. Yushkevich, P.A., Zhang, H., Simon, T.J., Gee, J.C.: Structure-specific statistical mapping of white matter tracts. NeuroImage 41(2), 448-461 (2008) 